\title{
Therapeutic Effects of Intra-articular Botulinum Toxin Type A in Knee Osteoarthritis
}

\author{
Wang-Sheng Lin and Chen-Liang Chou* \\ Department of Physical Medicine and Rehabilitation, Taipei Veterans General Hospital, Taipei, Taiwan
}

*Corresponding author: Chen-Liang Chou, Department of Physical Medicine and Rehabilitation, National Yang-Ming University School of Medicine, Taipei, Taiwan, Tel: +886-2-28757296; E-mail: cl_chou@vghtpe.gov.tw

Rec date: June 27, 2016; Acc date: June 28, 2016; Pub date: June 30, 2016

Copyright: ( 2016 Lin WS et al. This is an open-access article distributed under the terms of the Creative Commons Attribution License, which permits unrestricted use, distribution, and reproduction in any medium, provided the original author and source are credited.

Keywords: Botulinum toxin; Knee osteoarthritis

\section{Knee Osteoarthritis}

Knee osteoarthritis (KOA) is an intractable and devastating consequence of degeneration that results in tremendous impact on daily activities. Painful disabling KOA occurs in more than $10 \%$ of people who are over 55 years old. Those who are severely disabled account for up to $25 \%$ of aging people and $\mathrm{KOA}$ is a major cause of total knee replacement [1-3]. Current osteoarthritis therapies largely rely on rest, weight loss, bracing and assistive devices, physical modalities, therapeutic exercises, and pharmacological interventions [4] which are unsatisfactory for the majority of severe disabled patients, who are left with ambulation deficit despite vigorous treatment intervention. Although numerous patients can be treated with surgery, some of them are not good candidates due to multiple co-morbidities. For these reasons, intra-articular (IA) treatments that reduce chronic joint pain and improve function is the long-term effective and safe alternative options. In a number of recent studies, pain intensity and functional performance resulted from osteoarthritis has been improved after IA injection of botulinum toxin type A (BoNT-A).

The Botulinum neurotoxin has been comprehensively studied for its muscle-paralyzing effect by proteolysis of membrane-associated proteins inhibiting of the exocytotic release of acetylcholine, thereby blocking neurotransmission from motor nerve terminals. However, as the growing body of research has demonstrated, IA BoNT-A injection applied to the painful joints can successfully improve symptoms and function by anti-nociceptive effect [5-9]. The mechanism of pain modulation after IA BoNT-A injection by means of two very different strategies by direct inhibition of nociceptive neuropeptides release such as substance $P$, calcitonin gene related peptide (cGRP), glutamate and the expression of the transient receptor potential vanilloid 1 at the primary sensory fibers, leading to a reduction of peripheral sensitization; by indirect inhibition of central sensitization associated with neuropathic pain through the reduction of the se and blocked of substance $P$ and cGRP secretion within central nerve system [10-12].

The role of botulinum toxin type A in the clinical management is now considered as part of an established option for chronic advanced arthritis or osteoarthritis if a patient has been refractory to conservative treatments, failed to corticosteroid or hyaluronic acid IA injection and unable to undergo joint surgical intervention. As compared with conventional therapy, IA BoNT-A injection has significant therapeutic effect on pain processing and functional recovery [13]. The pilot study provides evidence that BoNT-A compared to IA corticosteroid achieves a significant and persistent pain modulating effect [6]. The following study further confirmed that the IA BoNT-A compared to education only yielded favorable outcomes that were of considerable longevity, at least 6-month period [14]. Another study demonstrates that IA BoNT-A in patients with chronic painful total knee arthroplasty, providing short-term therapeutic effect, indicating IA BoNT-A not only play a role on alleviating KOA symptoms before surgery but also post-operation pain control. Although there is a lack of head-to-head randomized trials to compared IA BoNT-A and IA hyaluronate in patients with knee osteoarthritis, but the efficacy was assessed in patients with unilateral ankle osteoarthritis. The study provides encouraging results related to pain relief and functional recovery by both interventions without statistically significant intergroup difference, these effects initiating at 2 weeks and last for half year [9].

The dose-dependent effect of BoNT-A has not yet been proved due to only single research observed the difference [6]. Further studies should focus on the response rate of different doses. There are two subtypes of botulinum neurotoxin are available for clinical practice which are serotype A and serotype B. Though BoNT-B is as clinically effective as BoNT-A, BoNT-A is more widely used in management of pain and spasticity. That is possible due to more side effects and shorter more efficacy after BoNT-B injection [15]. The related literature review on this issue revealed no severe adverse events among all studies [13].

Recent advances in the use of botulinum toxin type A have highlighted the benefit of anti-nociceptive effect in osteoarthritis. Such pain modulation consolidates induced peripheral inhibition and central desensitization effects when it is applied by intra-articular injection. This innovative and safe method could be applied as a complementary therapy performed in conjunction with traditional KOA treatment.

\section{References}

1. Peat G, McCarney R, Croft P (2001) Knee pain and osteoarthritis in older adults: a review of community burden and current use of primary health care. Annals of the rheumatic diseases 60: 91-97.

2. Zhang Y, Jordan JM (2010) Epidemiology of osteoarthritis. Clinics in geriatric medicine 26: 355-369.

3. Woolf AD, Pfleger B (2003) Burden of major musculoskeletal conditions. Bulletin of the World Health Organization 81: 646-656.

4. Hochberg MC, Altman RD, April KT, Benkhalti M, Guyatt G, et al. (2012) American College of Rheumatology recommendations for the use of nonpharmacologic and pharmacologic therapies in osteoarthritis of the hand, hip, and knee. Arthritis care \& research 64: 465-474.

5. Mahowald ML, Singh JA, Dykstra D (2006) Long term effects of intraarticular botulinum toxin A for refractory joint pain. Neurotoxicity research 9: 179-188.

6. Boon AJ, Smith J, Dahm DL, Sorenson EJ, Larson DR, et al. (2010) Efficacy of intra-articular botulinum toxin type A in painful knee osteoarthritis: a 
Citation: Lin WS, Chen CL (2016) Therapeutic Effects of Intra-articular Botulinum Toxin Type A in Knee Osteoarthritis. J Arthritis 5: e112. doi:

Page 2 of 2

pilot study. PM and R: the journal of injury, function, and rehabilitation 2 268-276.

7. Chou CL, Lee SH, Lu SY, Tsai KL, Ho CY, et al. (2010) Therapeutic Effects of Intra-articular Botulinum Neurotoxin in Advanced Knee Osteoarthritis. Journal of the Chinese Medical Association 73: 573-580.

8. Marchini C, Acler M, Bolognari MA, Causero A, Volpe D, et al. (2010) Efficacy of botulinum toxin type A treatment of functional impairment of degenerative hip joint: Preliminary results. Journal of rehabilitation medicine 42: 691-693.

9. Sun SF, Hsu CW, Lin HS, Chou YJ, Chen JY, et al. (2014) Efficacy of intraarticular botulinum toxin $\mathrm{A}$ and intraarticular hyaluronate plus rehabilitation exercise in patients with unilateral ankle osteoarthritis: a randomized controlled trial. Journal of foot and ankle research 7: 9.

10. Singer BJ, Silbert BI, Silbert PL, Singer KP (2015) The Role of Botulinum Toxin Type A in the Clinical Management of Refractory Anterior Knee Pain Toxins 7: 3388-3404.
11. Pellett S, Yaksh TL, Ramachandran R (2015) Current status and future directions of botulinum neurotoxins for targeting pain processing. Toxins (Basel) 7: 4519-4563.

12. Oh HM, Chung ME (2015) Botulinum Toxin for Neuropathic Pain: A Review of the Literature. Toxins (Basel) 7: 3127-3154.

13. Wu T, Song HX, Dong Y, Ye Y, Li JH (2016) Intra-articular injections of botulinum toxin a for refractory joint pain: A systematic review and metaanalysis. Clin Rehabil pii: 0269215516644951.

14. Hsieh LF, Wu CW, Chou CC, Yang SW, Wu SH, et al. (2016) Effects of Botulinum Toxin Landmark-Guided Intra-articular Injection in Subjects with Knee Osteoarthritis. PM R pii: S1934-1482.

15. Bentivoglio AR, Grande AD, Petracca M, Ialongo T, Ricciardi L (2015) Clinical differences between botulinum neurotoxin type A and B. Toxicon 107: 77-84. 